\title{
Complete genome sequence of Bacillus coagulans CACC834 isolated from canine
}

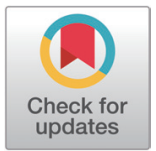

Received: Jun 21, 2021

Revised: Sep 15, 2021

Accepted: Sep 25, 2021

*Corresponding author

Yangseon Kim

Department of Research

and Development, Center for

Industrialization of Agricultural and

Livestock Microorganisms, Jeongeup

56212 , Korea.

Tel: +82-63-536-6712

E-mail: yangseon@cialm.or.kr

Copyright (c) 2021 Korean Society of Animal Sciences and Technology.

This is an Open Access article distributed under the terms of the Creative Commons Attribution Non-Commercial License (http:// creativecommons.org/licenses/by$\mathrm{nc} / 4.0 /$ ) which permits unrestricted non-commercial use, distribution, and reproduction in any medium, provided the original work is properly cited.

ORCID

Jung-Ae Kim

https://orcid.org/0000-0002-0694-477X

Dae-Hyuk Kim

https://orcid.org/0000-0002-9948-5313

Yangseon Kim

https://orcid.org/0000-0002-8285-3407

Competing interests

No potential conflict of interest relevant to this article was reported.

Funding sources

This research was supported by the Strategic Initiative for Microbiomes in Agriculture and Food grant(no.918002-4) and Ministry of Agriculture, Food and Rural Affairs (no.321094-2), Korea.

\author{
Jung-Ae Kim ${ }^{1,2}$, Dae-Hyuk Kim ${ }^{1,3}$ and Yangseon Kim* \\ ${ }^{1}$ Department of Research and Development, Center for Industrialization of Agricultural and Livestock \\ Microorganisms, Jeongeup 56212, Korea \\ ${ }^{2}$ Department of Bioactive Material Science, Jeonbuk National University, Jeonju 54896, Korea \\ ${ }^{3}$ Department of Molecular Biology, Department of Bioactive Material Science, Institute for Molecular \\ Biology and Genetics, Jeonbuk National University, Jeonju 54896, Korea
}

\begin{abstract}
Bacillus coagulans CACC 834 was isolated from canine feces, and its potential probiotic properties were characterized by functional genome analysis. Whole-genome sequencing of $B$. coagulans CACC 834 was performed using the PacBio RSII platforms. The complete genome assembly consisted of one circular chromosome (3.1 Mb) with guanine $(\mathrm{G})+\mathrm{cy}-$ tosine (C) content of $47.1 \%$. Annotation revealed 3,181 protein-coding sequences (CDSs), 30 rRNAs, and 83 tRNAs. Gene associated $11 \%$ of the genes were involved in replication, recombination, and repair. We also annotated various stress-related, acid resistance, bile salt resistance and adhesion-related domains in this strain, which likely provide support in exerting probiotic action by survival under gastrointestinal tract. These results add to our comprehensive understanding of $B$. coagulans and suggest potential mammal-related industrial applications.
\end{abstract}

Keywords: Bacillus coagulans, Canine, Whole-genome sequencing

Bacillus coagulans strains are gram-positive, spore-forming, and produce lactic acid, which possesses the capacity to balance intestinal gut microbiota, ultimately promoting the growth of animals and improving immunity [1,2]. In addition, $B$. coagulans is resistant to high temperatures because of its probiotic activity, modulates and strengthens the immune system to protect against infections, and minimizes inflammation-related tissue damage [3].

We isolated B. coagulans CACC 834 (KACC 22145) from the feces of a female 3-year-old Boston terrier in Korea. The samples were incubated under anaerobic conditions at $37^{\circ} \mathrm{C}$ [4].

Whole-genome sequencing was performed using the Pacific Biosciences (PacBio) RS II Single Molecule Real Time (SMRT) platform with a $20 \mathrm{~kb}$ SMRTbell ${ }^{\mathrm{TM}}$ template library at ChunLab. The reads were assembled using the PacBio SMAR Analysis 2.3.0. [5].

The sequences were annotated using the combined results of the automatic National Center for Biotechnology Information (NCBI) Prokaryotic Genome Annotation Pipeline (PGAP) and Rapid Annotations using Subsystems Technology (RAST) [6].

The complete genome of B. coagulans CACC 834 was composed of a 3,077,319 bp circular chromosome with $47.1 \%$ guanine $(\mathrm{G})+$ cytosine $(\mathrm{C})$ content. The genome contained 3,181 protein- 
Acknowledgements Not applicable.

Availability of data and material Upon reasonable request, the datasets of this study can be available from the corresponding author.

\section{Authors' contributions} Conceptualization: Kim Y.

Data curation: Kim JA.

Formal analysis: Kim JA.

Methodology: Kim JA.

Software: Kim JA.

Validation: Kim JA, Kim DH

Investigation: Kim Y.

Writing - original draft: Kim Y.

Writing - review \& editing: Kim JA, Kim DH, Kim Y.

Ethics approval and consent to participate This article does not require IRB/IACUC approval because there are no human and animal participants. coding sequences (CDS), $30 \mathrm{rRNAs}$, and $83 \mathrm{tRNAs}$ (Table 1). The genome features of $B$. coagulans CACC 834 are summarized in Fig. 1. Among these CDS, 2,909 genes were classified into 20 clusters of orthologous groups (COG) functional categories (Fig. 1B). More than $41 \%$ of genes were involved in transport and metabolism, including amino acid, carbohydrate, inorganic ion, nucleotide, coenzyme, and lipid. Interestingly, $11 \%$ of the genes were involved in replication, recombination, and repair (Fig. 1B).

The genome of $B$. coagulans CACC 834 possessed bsp20, hsp60, dnaK, dnaJ and grpE operons, encoding enzymes and proteins for heat shock. Also, B. coagulans CACC 834 has heat shock induced genes, such as $\operatorname{ll} B, \operatorname{cl} p P, m c s B$, and repair-related genes, such as $\operatorname{rec} A$ and $u v r A B C$. The expression of these genes is expected to help promote cell recovery from heat shock by limiting damage caused by stress [7-9]. Furthermore, B. coagulans CACC 834 carried genes known to be involved in lactate synthesis, adhesion, acid resistance, and bile resistance (Table 2).

This study on complete genome sequence of $B$. coagulans CACC 834 may increase our

Table 1. Genome overview of Bacillus coagulas CACC834

\begin{tabular}{|c|c|}
\hline Feature & Values \\
\hline Genome size (bp) & $3,077,319$ \\
\hline No. of contigs & 1 \\
\hline GC content & $47.1 \%$ \\
\hline Protein-coding genes (CDSs) & 684 \\
\hline rRNA & 30 \\
\hline tRNA & 83 \\
\hline plasmid & 0 \\
\hline Genbank Acession No. & СР076597 \\
\hline
\end{tabular}

A

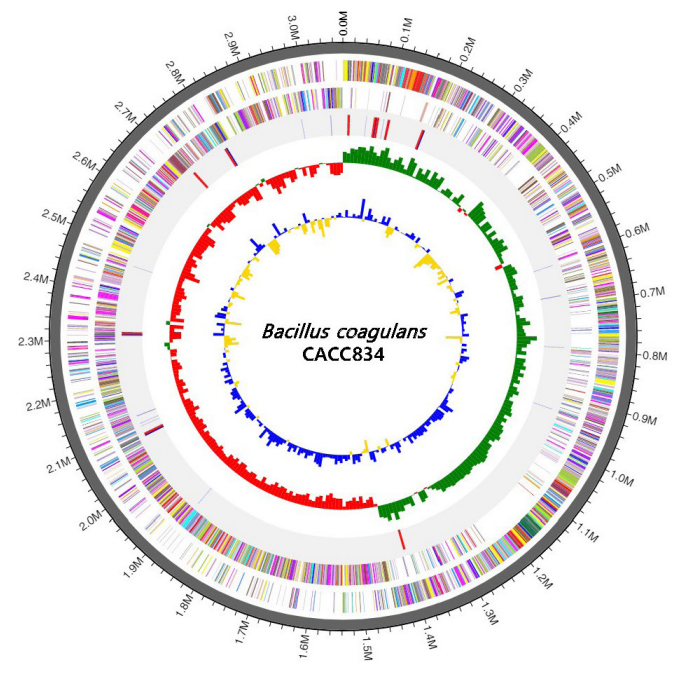

B

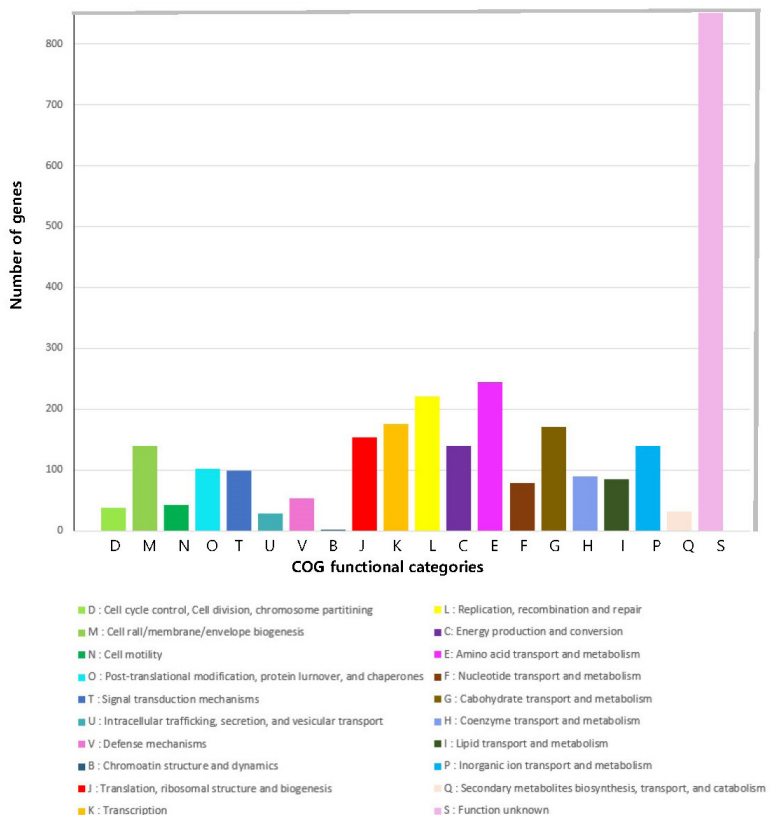

Fig. 1. Genome features of Bacillus coagulans CACC834. (A) Circular genome maps of B. coagulans CACC834 chromosome. Circles from the outside to the center denote rRNA and tRNA gene, reverse strand CDS, forward strand CDS, GC skew, and GC content. (B) Genome number of COG categorie. CDS, protein-coding genes; COG, clusters of orthologous group; G, guanine; C, cytosine. 
Table 2. Predicted genes involved in probiotic potency in B. coagulans CACC834

\begin{tabular}{|c|c|c|c|c|}
\hline \multirow{2}{*}{ Predicted function } & \multicolumn{4}{|c|}{ B. coagulans CACC 834} \\
\hline & Predicted genes & Start position & End position & Length (bp) \\
\hline Lactate synthesis & Idh & $1,433,259$ & $1,434,242$ & 984 \\
\hline \multirow[t]{2}{*}{ Adhesion protein } & EpsD & 393,741 & 394,838 & 1,098 \\
\hline & FliD & 724,445 & 726,531 & 2,088 \\
\hline \multicolumn{5}{|l|}{ Acid resistance-related } \\
\hline \multirow[t]{8}{*}{ Protection or repair of macromolecules } & dnaK & $1,737,678$ & $1,737,546$ & 1,827 \\
\hline & dnaJ & $1,736,410$ & $1,737,546$ & 1,137 \\
\hline & $\operatorname{grp} E$ & $1,739,540$ & $1,740,202$ & 663 \\
\hline & recA & 125,046 & $1,226,113$ & 1,068 \\
\hline & uvrABC & 765,135 & 767,135 & 2,001 \\
\hline & $c / p B$ & 108,312 & 110,762 & 2,451 \\
\hline & $c / p P$ & 793,689 & 794,279 & 591 \\
\hline & $m c s B$ & 108,312 & 110,762 & 2,451 \\
\hline \multirow[t]{4}{*}{ Fatty acid synthesis } & $f a b F$ & 870,816 & 871,754 & 1,242 \\
\hline & $\mathrm{fabH}$ & 871,824 & 873,065 & 939 \\
\hline & $f a d D$ & 260,088 & 261,242 & 1,155 \\
\hline & fabl & 903,131 & 903,910 & 780 \\
\hline Acid shock response & aspS & $1,804,625$ & 180,696 & 1,773 \\
\hline Metabolic rearrangements & als $D$ & $2,318,874$ & $2,319,623$ & 750 \\
\hline Gylcine betain $A B C$ transport system & opuCC & 467,919 & 468,833 & 915 \\
\hline Bile salt resistance & bass & $1,090,675$ & $1,091,145$ & 471 \\
\hline
\end{tabular}

understanding of the probiotic effects in host healthcare and extend its potential application as an industrial strain.

The complete genome of $B$. coagulans strain CACC 834 determined in this study has been deposited in the NCBI GenBank database under accession number CP076597.

\section{REFERENCES}

1. Su F, Xu P. Genomic analysis of thermophilic Bacillus coagulans strains: efficient producers for platform bio-chemicals. Sci Rep. 2014;4:3926. https://doi.org/10.1038/srep03926

2. Zhou Y, Zeng Z, Xu Y, Ying J, Wang B, Majeed M, et al. Application of Bacillus coagulans in animal husbandry and its underlying mechanisms. Animals. 2020;10:454. https://doi. org/10.3390/ani10030454

3. Konuray G, Erginkaya Z. Potential use of Bacillus coagulans in the food industry. Foods. 2018;7:92. https://doi.org/10.3390/foods7060092

4. Jang HJ, Son S, Kim JA, Jung MY, Choi Y, Kim DH, et al. Characterization and functional test of canine probiotics. Front Microbiol. 2021;12:625562. https://doi.org/10.3389/ fmicb.2021.625562

5. Lee JE, Heo S, Kim GB. Complete genome sequence of Streptococcus hyointestinalis B19, a strain producing bacteriocin, isolated from chicken feces. J Anim Sci Technol. 2020;62:420-2. https://doi.org/10.5187/jast.2020.62.3.420

6. Aziz RK, Bartels D, Best AA, DeJongh M, Disz T, Edwards RA, et al. The RAST Server: rapid annotations using subsystems technology. BMC Genomics. 2008;9:75. https://doi. org/10.1186/1471-2164-9-75 
7. Palop A, Sala FJ, Condon S. Occurrence of a highly heat-sensitive spore subpopulation of Bacillus coagulans STCC 4522 and its conversion to a more heat-stable form. Appl Environ Microbiol. 1997;63:2246-51. https://doi.org/10.1128/aem.63.6.2246-2251.1997

8. Guzzo J. Biotechnical applications of small heat shock proteins from bacteria. Int J Biochem Cell Biol. 2012;44:1698-705. https://doi.org/10.1016/j.biocel.2012.06.007

9. Li P, Tian W, Jiang Z, Liang Z, Wu X, Du B. Genomic characterization and probiotic potency of Bacillus sp. DU-106, a highly effective producer of L-lactic acid isolated from fermented yogurt. Front Microbiol. 2018;9:2216. https://doi.org/10.3389/fmicb.2018.02216 